02

\title{
Внутризонные осцилляции Раби с участием фотонов и акустических фононов
}

\author{
(C) Е.Ю. Перлин, А.В. Иванов \\ Университет ИТМО, \\ 199034 Санкт-Петербург, Россия \\ e-mail: eyperlin@bk.ru
}

Поступила в редакцию 15.12 .2020 г.

В окончательной редакции 15.12.2020 г.

Принята к публикации 24.12.2020 г.

Получены вероятности $W_{\text {ехс }}$ внутризонных переходов электронов с участием фотонов и продольных акустических фононов в ковалентном кристалле. Показано, что в случае предпробойных интенсивностей света могут иметь место специфические электрон-фононные осцилляции Раби с частотой $\Omega_{\mathrm{R}}^{\text {(eff) }}$, на которую влияют процессы высших порядков по полю световой волны. Как и в ранее рассмотренном случае процессов с участием оптических фононов, поглощение света идет только до времени достижения первого максимума $\tau_{1}$ на зависимости $W_{\text {exс }}(t)$, где $t-$ время, прошедшее с начала действия лазерного импульса.

Ключевые слова: внутризонное поглощение света, осцилляции Раби, деформационный потенциал, ковалентные кристаллы, предпробойные интенсивности света.

DOI: $10.21883 / \mathrm{OS} .2021 .04 .50780 .302-20$

\section{1. Введение}

В предыдущей работе авторов [1] была развита теория поглощения мощного лазерного излучения свободными электронами на непрямых внутризонных переходах с участием фотонов и продольных оптических (LO) фононов. Были получены относительно простые аналитиче-

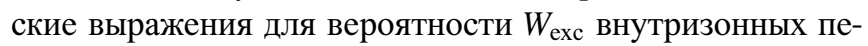
реходов с изменением энергии электрона на $\hbar\left(\omega \pm \omega_{l}\right)$, где $\omega_{l}$ - частота LO-фононов, участвующих в процессе, а $\omega$ - частота лазерного излучения видимого либо ближнего ИК диапазона. Для расчетов в работе [1] было использовано модифицированное для случая переходов с участием фотонов и фононов резонансное приближение, а также техника „распутывания“ экспоненциальных операторов. Основной результат работы [1] состоит в том, что в случае очень высоких интенсивностей лазерного излучения имеют место специфические электронфононные осцилляции Раби с частотой $\Omega_{\mathrm{R}}$, а поглощение света идет только до времени достижения первого максимума $\tau_{1}$ на зависимости $W_{\text {exс }}(t)$, где $t-$ время, прошедшее с начала действия лазерного импульса. В [1] показано также, что на $W_{\text {exc }}, \tau_{1}$ и $\Omega_{\mathrm{R}}$ существенным образом влияют процессы высоких порядков по полю световой волны.

В работе [1] был рассмотрен лишь один механизм взаимодействия электронов с колебательной подсистемой кристалла, а именно полярное взаимодействие электронов c LO-фононами, описываемое гамильтонианом Фрёлиха. В настоящей работе, которая является продолжением работы [1], будет показано, что внутризонные фотон-фононные осцилляции Раби могут иметь место и при взаимодействии сильного света с ковалентными кристаллами, когда в непрямых внутризонных переходах могут принимать участие лишь продольные акустические (LA) фононы.

\section{2. Вероятность возбуждения электронной системы}

Расчет вероятности внутризонного возбуждения свободных электронов $W_{\text {ехс }}$ выполняется тем же методом и в рамках тех же приближений, что и в работе [1]. Отличие состоит в том, что вместо гамильтониана Фрёлиха (см. [1], формулы (7) и (8)), описывающего полярное взаимодействие электронов c LO-фононами, здесь мы используем гамильтониан взаимодействия электронов с LA-фононами [2]:

$$
H_{\mathrm{e}-\mathrm{phon}}^{\prime}=\frac{1}{\sqrt{N}} \sum_{\mathbf{k}, \mathbf{q}, s} F_{S}(\mathbf{q}) a_{\mathbf{k}+\mathbf{q}}^{+} a_{\mathbf{k}}\left(b_{\mathbf{q} s}-b_{-\mathbf{q} s}^{+}\right),
$$

где

$$
F_{s}(\mathbf{q})=-i D \sqrt{\frac{\hbar|\mathbf{q}|}{2 M c_{s}}}
$$

$a_{\mathbf{k}}^{+}$и $a_{\mathbf{q}}-$ операторы рождения и уничтожения электронов в зоне проводимости $c$ с волновым вектором $\mathbf{k}, b_{\mathbf{q}}^{+}$ и $b_{\mathbf{q}}$ - операторы рождения и уничтожения LA-фононов с волновым вектором q, $D$ - константа деформационного потенциала, $M$ - масса элементарной ячейки, $N$ - число элементарных ячеек, $c_{s}$ - скорость звука в кристалле. Метод деформационного потенциала для описания взаимодействия электронов с акустическими фононами был впервые предложен в работах $[3,4]$. 
В результате расчетов с использованием (1) и (2) получим следующее выражение для вероятности перехода из состояний с волновыми векторами электронов $\mathbf{k}$ вблизи дна зоны проводимости в состояния с волновыми векторами $\mathbf{k}+\mathbf{q}$ :

$$
\begin{aligned}
& W_{\text {exc }}(t)=\frac{1}{2} \int_{0}^{\infty} f(k) d k \int_{0}^{\infty} \sum_{s=1}^{2} \frac{\left|P_{s}(k)\right|^{2}}{\left|P_{s}(k)\right|^{2}+\left[\frac{1}{2} \delta_{s}(q)\right]^{2}} \\
& \times \sin ^{2}\left\{\sqrt{\left|P_{s}(k)\right|^{2}+\left[\frac{1}{2} \delta_{s}(q)\right]^{2} t}\right\} q^{3} d q,
\end{aligned}
$$

где

$$
\begin{gathered}
f(k)=\frac{\hbar^{9 / 2} k}{4 \pi^{2} m_{\mathrm{c}}^{3}}\left(\frac{\pi}{k_{\mathrm{B}} T_{\mathrm{eff}} \omega}\right)^{3 / 2} \exp \left[-\frac{\hbar^{2} k^{2}}{2 m_{c} k_{\mathrm{B}} T_{\mathrm{eff}}}\right], \\
\delta_{1,2}(q)=\frac{\hbar q^{2}}{2 m_{\mathrm{c}}}-\omega \mp q c_{\mathrm{s}}, \\
T_{\mathrm{eff}}=T_{0}+\Delta T, \Delta T=\frac{8 \pi j_{\omega}}{3 c k_{\mathrm{B}} m_{c} \omega^{2}},
\end{gathered}
$$

$m_{\mathrm{c}}$ - эффективная масса электрона, $k_{\mathrm{B}}-$ постоянная Больцмана, $T_{0}$ - температура решетки, $q c_{s}=\omega_{a}-$ частоты акустических фононов с волновым числом $q$,

$$
\begin{aligned}
& P_{1,2}^{2}(k)=\frac{2^{1 / 2} D^{2} \vartheta^{2}\left(m_{\mathrm{c}} \omega\right)^{3 / 2} k}{\pi^{2} \hbar^{5 / 2} \rho c_{s}} \\
& \times\left\{\begin{array}{l}
{\left[e^{\hbar c_{s} q_{0} /\left(k_{\mathrm{B}} T_{0}\right)}-1\right]^{-1}} \\
\left\{1+\left[e^{\left.\hbar c_{\mathrm{s} q_{0} /\left(k_{\mathrm{B}} T_{0}\right.}\right)}-1\right]^{-1}\right\}^{-1}
\end{array}\right. \\
& \vartheta=J_{1}\left(\xi_{2}-\zeta_{1}\right), \zeta_{1,2}=\frac{2 e k_{1,2}}{n_{c} \omega^{2}} \sqrt{\frac{2 \pi j \omega}{c \sqrt{\varepsilon_{\infty}}}}, \\
& k_{1} \approx \frac{1}{\hbar} \sqrt{m_{c} k_{\mathrm{B}} T_{\mathrm{eff}}}, k_{2} \approx \sqrt{\frac{m_{c} \omega}{3 \hbar}}, \\
& q_{0}=\sqrt{2 m_{c} \omega / \hbar},
\end{aligned}
$$

где $\rho-$ плотность материала, $J_{n}(\xi)-$ функции Бесселя.

\section{3. Результаты расчетов}

На рис. 1 показаны зависимости вероятности внутризонного возбуждения электрона $W_{\mathrm{exc}}$ от времени $t$ с момента начала действия лазерного импульса, рассчитанные с помощью формул (3)-(8) при следующих значениях параметров: $m_{\mathrm{c}}=0.35 \mathrm{~m}, m-$ масса свободного электрона, $\varepsilon_{\infty}=11.5, D=7 \mathrm{eV}, \rho=2.33 \mathrm{~g} / \mathrm{cm}^{3}$, $c_{\mathrm{s}}=2.2 \cdot 10^{5} \mathrm{~cm} / \mathrm{s}, T_{0}=300 \mathrm{~K}$. Указанные значения параметров характерны для кристаллов типа Si. Для удобства сравнения полученных здесь результатов с результатами работы [1] графики на рис. 1 приводятся для тех же значений частот света $\omega$ интенсивностей $j_{\omega}$, что и на рис. 2 работы [1].

На рис. 2 показаны зависимости максимальных значений $W_{\max }$ вероятностей $W_{\text {exc }}(t)$ от интенсивности лазерного излучения $j_{\omega}$.
На рис. 3 показаны зависимости времени достижения первого максимума $\tau_{1}$ на зависимости $W_{\max }(t)$ от интенсивности $j_{\omega}$.

На рис. 4 показаны зависимости эффективных частот Раби $\Omega_{\mathrm{R}}^{\mathrm{eff}}$ от интенсивности света $j_{\omega}$. Как и работе $[1], \Omega_{\mathrm{R}}^{(\mathrm{eff})}$ определяется следующим образом: $\Omega_{\mathrm{R}}^{(\mathrm{eff})}=2 \pi /\left(\tau_{2}-\tau_{1}\right)$, где $\tau_{2}-$ время, за которое достигается второй максимум на кривой $W_{\text {exc }}(t)$.

На рис. 5 показаны зависимости максимальных значений $W_{\max }$ вероятностей $W_{\text {exc }}(t)$ от частоты света $\omega$.

\section{4. Обсуждение результатов}

Характер зависимостей, приведенных в предыдущем разделе, в основном такой же, как и в случае взаимодействия электронов c LO-фононами, рассмотренном в работе [1]. Тем не менее имеются некоторые отличия, которые мы обсудим в этом разделе. Осцилляции Раби в случае процессов с LA-фононами (рис. 1) более четко выражены и имеют заметно бо́льшую относительную амплитуду, чем в случае процессов с участием LO-фононов [1]. Эффективные частоты Раби $\Omega_{\mathrm{R}}^{(\mathrm{eff})}$ в области низких частот света $\left(\omega \approx 0.8 \cdot 10^{15} \mathrm{~s}^{-1}\right)$ меньше, чем в случае процессов с LO-фононами. C увеличением частоты света $\omega$ при фиксированной интенсивности $j \omega$ в случае процессов с LA-фононами $\Omega_{\mathrm{R}}^{(\mathrm{eff})}$ убывает медленнее, чем для процессов с LO-фононами.

На кривых $W_{\max }\left(j_{\omega}\right)$ при фиксированных значениях $\omega$ (рис. 2) и на кривых $W_{\max }(\omega)$ при фиксированных $j_{\omega}$ (рис. 5) имеются значительно более выраженные по сравнению со случаем LO-фононов области, где $W_{\max }\left(j_{\omega}\right)$ падает с увеличением $j_{\omega}$, а $W_{\max }(\omega)$ растет с увеличением $\omega$. Как уже отмечалось в [1], наличие таких областей связано с процессами высоких порядков по полю световой волны.

Указанные выше различия между приведенными зависимостями в случаях процессов с участием акустических либо оптических фононов связаны в первую очередь с различиями в коэффициентных функциях $F_{s}(\mathbf{q})$ в операторах электрон-фононного взаимодействия. В случае взаимодействия с LO-фононами, описываемого гамильтонианом Фрёлиха (формула (7) в [1]), $F_{s} \propto q^{-1}$, тогда как для деформационного потенциала, описывающего взаимодействие c LA-фононами (формулы (1) и (2) настоящей работы), $F_{s} \propto q^{1 / 2}$. Поскольку актуальные значения $q \approx q_{0} \propto \omega^{1 / 2}$ (см. формулу (8)), с ростом частоты света $\omega$ соотношение между величинами $W_{\max }$, $\Omega_{\mathrm{R}}^{\mathrm{eff}}, \tau_{1}^{-1}$ для обоих случаев меняется в пользу процессов с участием LA-фононов.

В целом при выбранных в настоящей работе значениях параметров кристалла, близких к их значениям в $\mathrm{Si}$, и в работе $[1]$, близких к $\mathrm{ZnS}$, значения $W_{\max }$, $\tau_{1}, \Omega_{\mathrm{R}}^{\text {(eff) }}$ сопоставимы. При одновременном действии механизмов, описанных [1] и в настоящей работе, воз- 

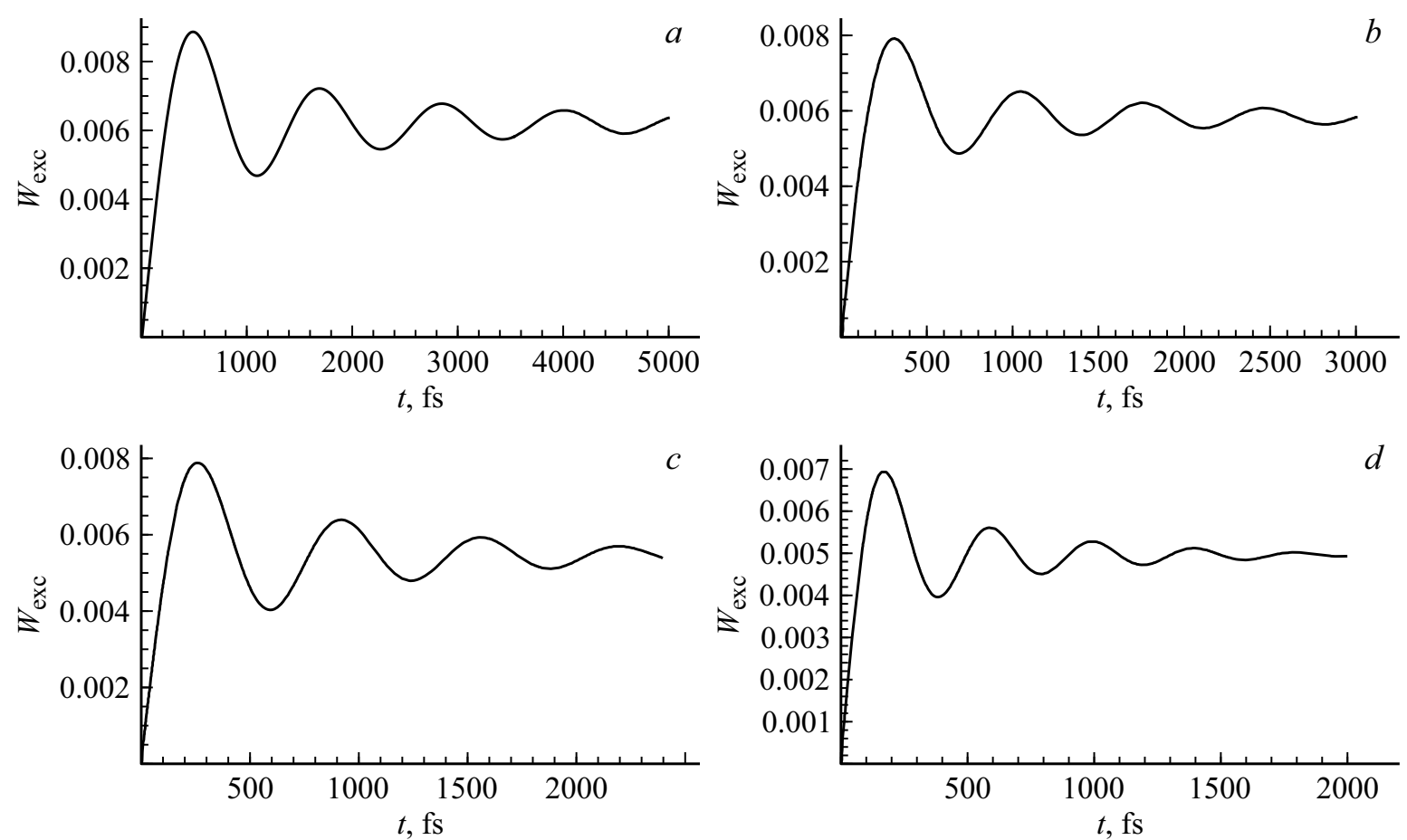

Рис. 1. Зависимости вероятности возбуждения электронов $W_{\mathrm{exc}}$ от времени $t$ с начала действия светового импульca: (a) $j_{\omega}=30 \mathrm{GW} / \mathrm{cm}^{2}, \omega=0.7 \cdot 10^{15} \mathrm{~s}^{-1}$, (b) $j_{\omega}=80 \mathrm{GW} / \mathrm{cm}^{2}, \omega=0.7 \cdot 10^{15} \mathrm{~s}^{-1}$, (c) $j_{\omega}=400 \mathrm{GW} / \mathrm{cm}^{2}, \omega=1.78 \cdot 10^{15} \mathrm{~s}^{-1}$, (d) $j_{\omega}=1 \mathrm{TW} / \mathrm{cm}^{2}, \omega=1.78 \cdot 10^{15} \mathrm{~s}^{-1}$.
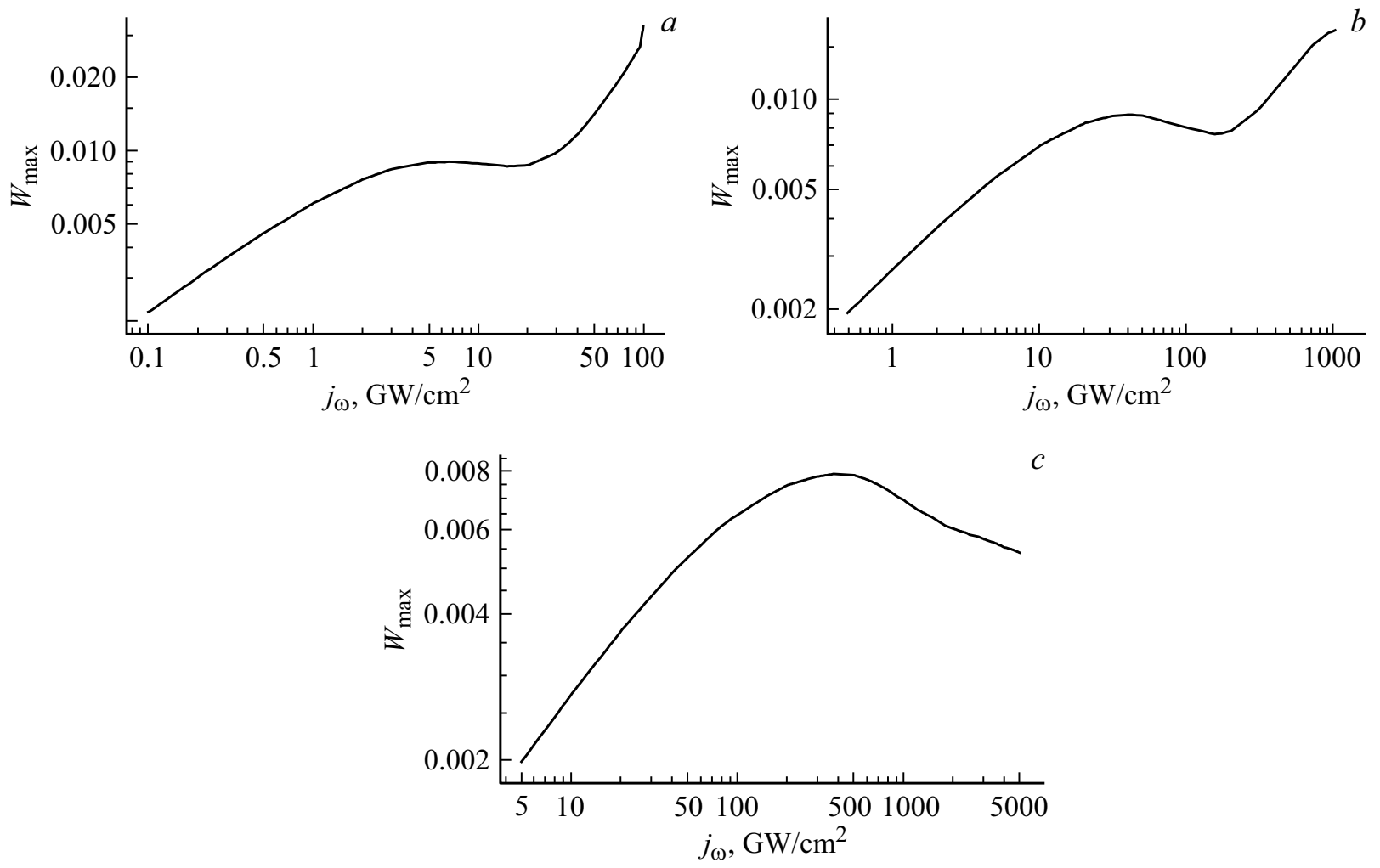

Рис. 2. Зависимости максимальных значений $W_{\max }$ от $j_{\omega}:(a) \omega=0.4 \cdot 10^{15} \mathrm{~s}^{-1},(b) \omega=0.8 \cdot 10^{15} \mathrm{~s}^{-1},(c) \omega=1.78 \cdot 10^{15} \mathrm{~s}^{-1}$. 

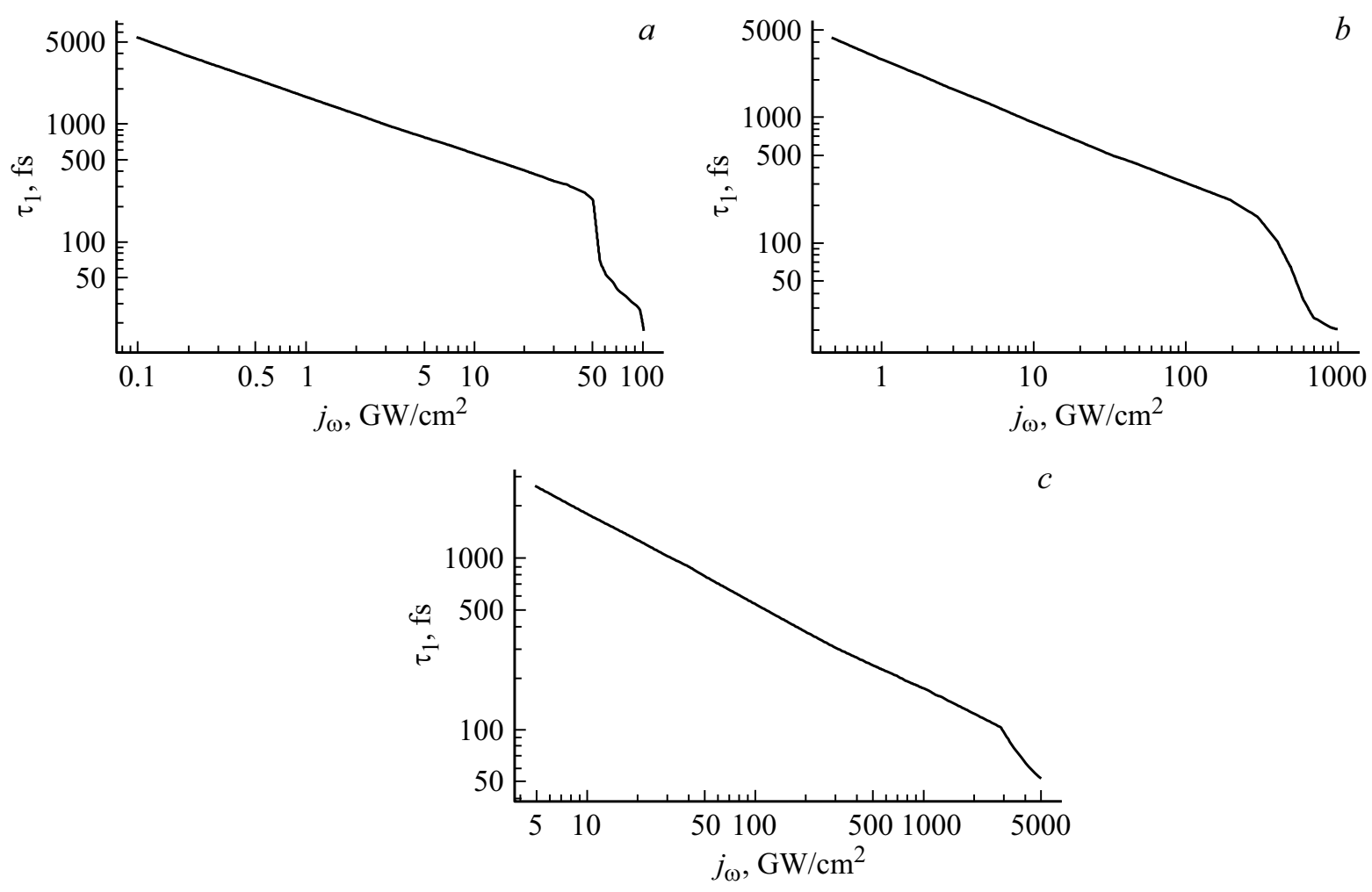

Рис. 3. Зависимости времени $\tau_{1}$ на кривых от $j_{\omega}:(a) \omega=0.4 \cdot 10^{15} \mathrm{~s}^{-1},(b) \omega=0.8 \cdot 10^{15} \mathrm{~s}^{-1},(c) \omega=1.78 \cdot 10^{15} \mathrm{~s}^{-1}$.
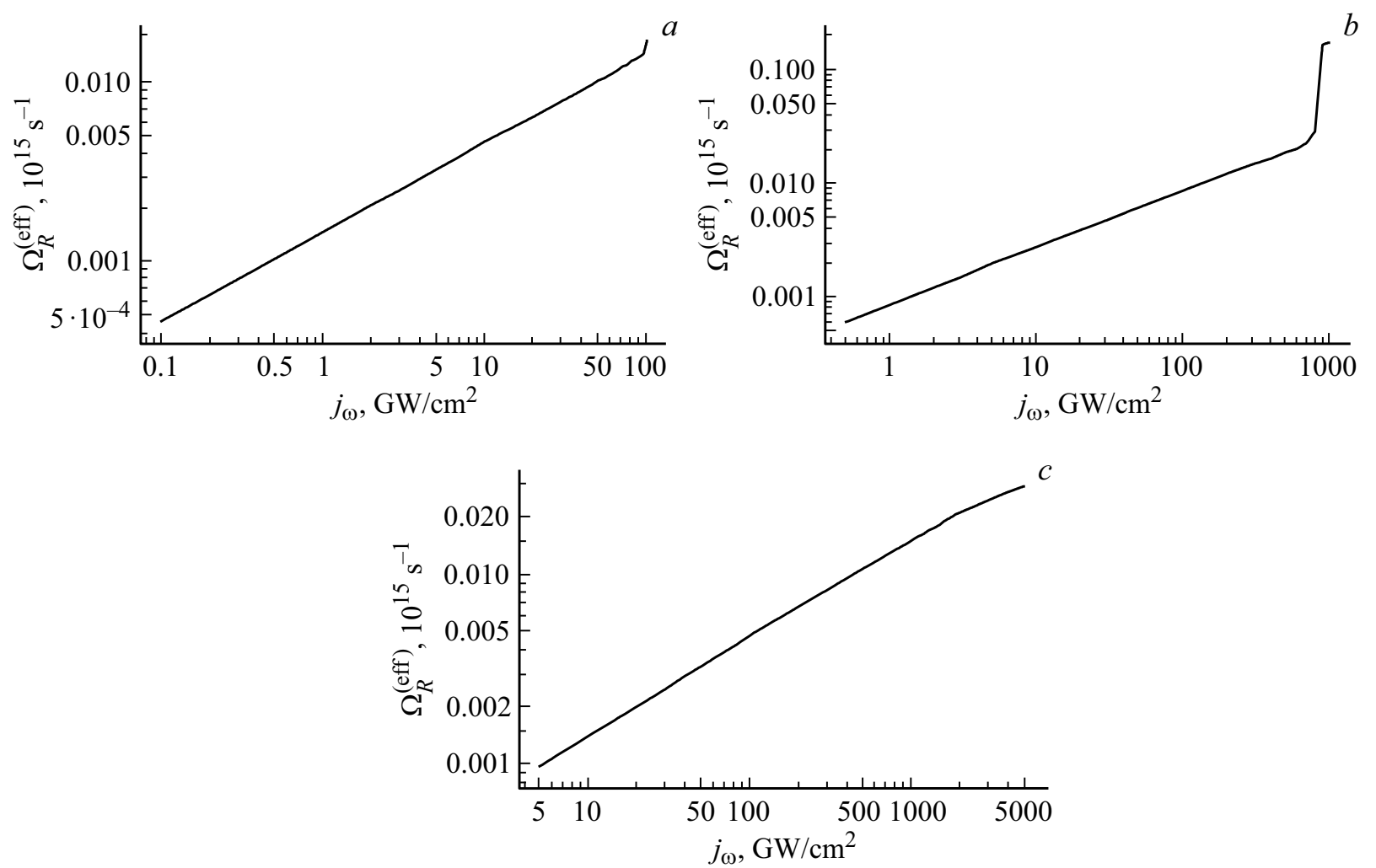

Рис. 4. Зависимости эффективных частот Раби $\Omega_{\mathrm{R}}^{(\mathrm{eff})}$ от интенсивности света $j_{\omega}$ : (a) $\omega=0.4 \cdot 10^{15} \mathrm{~s}^{-1},(b) \omega=0.8 \cdot 10^{15} \mathrm{~s}^{-1}$, (c) $\omega=1.78 \cdot 10^{15} \mathrm{~s}^{-1}$. 


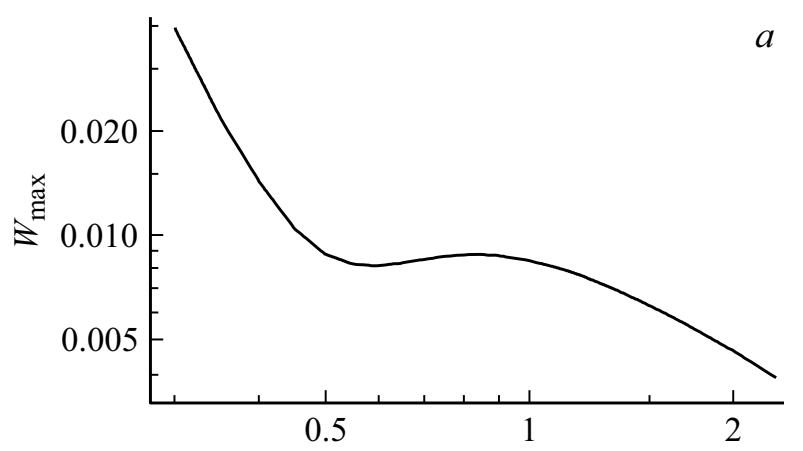

$\omega, 10^{15} \mathrm{~s}^{-1}$

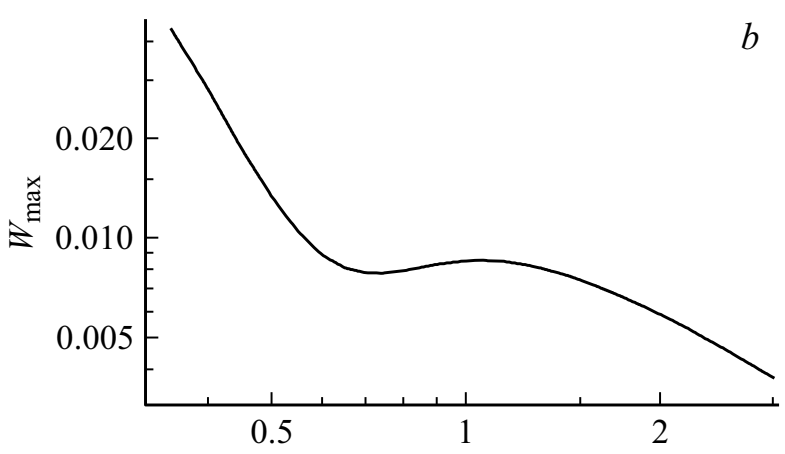

$\omega, 10^{15} \mathrm{~s}^{-1}$

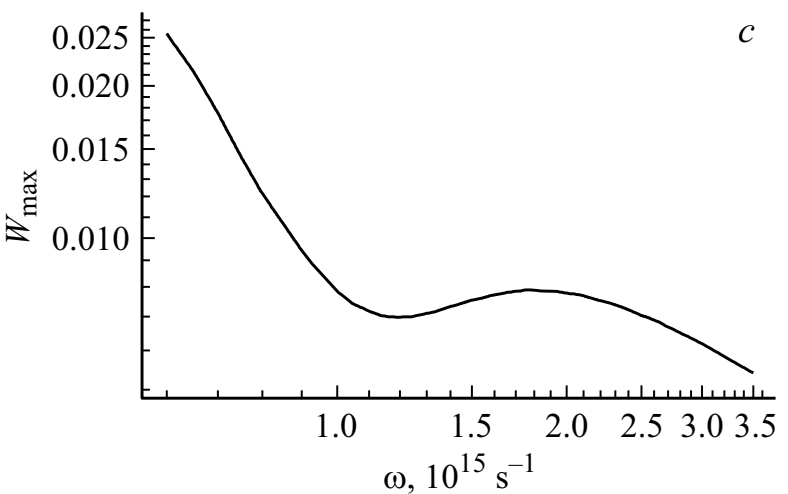

Рис. 5. Зависимости $W_{\max }$ от частоты света: $(a) j_{\omega}=50 \mathrm{GW} / \mathrm{cm}^{2},(b) j_{\omega}=100 \mathrm{GW} / \mathrm{cm}^{2},(c) j_{\omega}=500 \mathrm{GW} / \mathrm{cm}^{2}$.

можны ситуации, когда фотон-фононные осцилляции Раби усиливаются либо, наоборот, сглаживаются.

К некоторому сглаживанию осцилляций приводит также учет анизотропии зоны проводимости в ковалентных материалах. Остальные факторы, перечисленные в разд. 5 работы [1], актуальны и для случая, рассмотренного в настоящей работе.

Следует иметь в виду, что рассматриваемые в настоящей работе процессы могут влиять на пороговые для пробоя интенсивности света $j_{\mathrm{thr}}$, так как сначала происходит внутризонное поглощение, рассмотренное в настоящей работе, а уже затем пробой материала (см. также разд. 1 работы [1]). Типичные значения $j_{\text {th }}$ в очень чистых материалах составляют для пикосекундных и субпикосекундных лазерных импульсов в зависимости от частотного диапазона и структуры мод лазерного излучения $10^{13}-10^{14} \mathrm{~W} / \mathrm{cm}^{2}$. Максимальные значения интенсивностей $j_{\omega}$, использованные при расчете графиков на рис. $2-4$, вплотную приближаются к $j_{\text {thr. }}$.

\section{5. Заключение}

В работе рассмотрено возбуждение мощным лазерным излучением свободных электронов на непрямых внутризонных переходах с участием фотонов и LAфононов. Получено простое выражение для вероятности перехода $W_{\text {exс }}$ из состояний электрона вблизи дна зоны проводимости в состояния с энергией, большей на вели- чину светового кванта. Показано, что в этом случае, как и в рассмотренном ранее в работе [1] случае процессов с участием LO-фононов, имеют место специфические электрон-фононные осцилляции Раби, а поглощение света идет только до времени достижения первого максимума $\tau_{1}$ на зависимости $W_{\text {exc }}$ от времени $t$ с начала действия лазерного импульса. Получены нелинейные зависимости максимальных значений $W_{\text {exc }}$ от интенсивности света $j_{\omega}$ при фиксированной частоте света $\omega$ и от $\omega$ при фиксированном значении $j_{\omega}$. Получены также зависимости $\tau_{1}$ и частоты фотон-фононных осцилляций Раби $\Omega_{\mathrm{R}}^{\text {(eff) }}$ от $j \omega$ при нескольких значениях $\omega$. Показано, что процессы высоких порядков по полю световой волны в рассматриваемом диапазоне предпробойных интенсивностей света существенным образом влияют на $W_{\mathrm{exc}}, \tau_{1}$ и $\Omega_{\mathrm{R}}^{(\mathrm{eff})}$.

\section{Финансирование работы}

Работа выполнена при государственной поддержке ведущих университетов Российской Федерации (субсидия 08-08).

\section{Конфликт интересов}

Авторы заявляют, что у них нет конфликта интересов. 


\section{Список литературы}

[1] Перлин Е.Ю., Иванов А.В., Попов А.А. // Опт. и спектр. 2020. Т. 128. № 12. С. 1854. doi 10.21883/OS.2020.12.50321.212-20; Perlin E.Yu., Ivanov A.V., Popov A.A. // Opt. Spectrosc. 2020. V. 12. N 12. P. 1988. doi 10.1134/S0030400X20121005

[2] Bardeen J., Shockley W. // Phys. Rev. 1950. V. 72. N 1. P. 72. doi 10.1103/PhysRev.80.72

[3] Дейген М.Ф., Пекар С.И. // ЖЭТФ. 1951. Т. 21. № 7. С. 803.

[4] Давыдов А.С. Теория твердого тела. М.: Наука, 1976. 640 с.; Davydov A.S. Theory of Solids. M.: Nauka, 1980. 